\title{
European Networks Focus on Advanced Materials
}

The European Networks on Advanced Materials were established in 1987 to enhance scientific and technical cooperation between research teams from different countries. With the assistance of industrial and public institutions and with the support of the Council of Europe and the Commission of the European Communities, the European Materials Research Society is continuing to develop these networks. Eleven networks have established programs, and three are in the initial stages of development (see Table).

This article is part of a continuing series that focuses on the philosophies, aims and activities of the separate networks as described by their chairmen. Featured this month is Network 9 on Gallium Arsenide.

A brochure detailing all the networks is available from: P. Siffert, Chairman, European Materials Research Society, Centre de Recherches Nucléaires, 23, rue de Loess, F67037, Strasbourg, France; telephone 8828 65 43; fax 88280990.

\section{Network 9-Gallium Arsenide}

Chairmen: W. Wettling and H.S. Rupprecht, Frauenhofer Institut für Angewandte, Freiburg, West Germany.

With the steadily increasing demand for faster devices in information technology and data processing, new materials are being investigated which, based on their superior physical properties, allow operation of higher frequencies than devices based on silicon. The most promising of these materials are the III-V semiconductors and among them the GaAs and GaAlAs sys- tem. In the near future operating frequencies of 50 to $100 \mathrm{GHz}$ for analog devices are envisaged, corresponding to switching times of 10 to 5 picoseconds in a digital inverter stage.

Work in this network's field of materials research aims at improving all technologically important growth and deposition processes of $\mathrm{GaAs}$ active layers on semiinsulating substrates and of metal and dielectric films on GaAs. Special emphasis will be put on a better understanding of interfaces between $\mathrm{GaAs}$ and metals and dielectrics with the objective to develop advanced processing concepts for GaAs microelectronic and optoelectronic integrated circuits.

Laboratories participating in this network include: MMEC, Leuven, Belgium; Universite VII, Paris, France; UMIST, Manchester, United Kingdom; STC, Harlow, United Kingdom; Plessey, Caswell Towcester, United Kingdom; INSA de Lyon, Villeurbanne, France; University of Nottingham, United Kingdom; TH, Darmstadt, West Germany; ELAB, Trondheim, Netherlands; Thomson CSF, Massy, France; University, Paderborn, West Germany; Max-Planck-Institut, Stuttgart, West Germany; CNET, Bagneux, France; IAF-FHG, Freiburg, West Germany; Tech Universitat, Clausthal, West Germany; University, Strasbourg, France; RSRE, St. Malvern, United Kingdom; KFA, Julich, West Germany; IAF-FHG, Freiburg, West Germany; MASPEC 4, Parma, Italy; CNRS, Villeneuve, France.

\section{E-MRS Networks on Advanced Materials}

\begin{tabular}{|c|c|c|}
\hline $\begin{array}{l}\text { Network } \\
\text { Number }\end{array}$ & Theme & Group Leaders \\
\hline 1 & Laser chemistry & $\begin{array}{l}\text { I.W. Boyd (UK) } \\
\text { E.F. Krimmel (FRG) }\end{array}$ \\
\hline 2 & Solid state ionics & M. Balkanski (France) \\
\hline 3 & $\begin{array}{l}\text { Modeling of } \\
\text { solidification }\end{array}$ & $\begin{array}{l}\text { H. Fredriksson } \\
\text { (Sweden) }\end{array}$ \\
\hline 4 & $\begin{array}{l}\text { Metastable alloy } \\
\text { production }\end{array}$ & $\begin{array}{l}\text { J. Bottiger, B. Stritzker, } \\
\text { M. von Allmen } \\
\text { (Denmark, FRG, } \\
\text { Switzertand) }\end{array}$ \\
\hline 5 & $\begin{array}{l}\text { Microanalysis of } \\
\text { semiconductors }\end{array}$ & $\begin{array}{l}\text { E. Sirtl, A. Cullis } \\
\text { (FRG, UK) }\end{array}$ \\
\hline 6 & $\begin{array}{l}\text { High energy ion } \\
\text { implantation }\end{array}$ & G.G. Bentini (italy) \\
\hline 7 & $\begin{array}{l}\text { II-VI Te-based } \\
\text { semiconductors }\end{array}$ & R. Triboulet (France) \\
\hline 8 & Biomaterials & D. Muster (France) \\
\hline 9 & Gallium arsenide & $\begin{array}{l}\text { H.S. Rupprecht, } \\
\text { W. Wettling (FRG) }\end{array}$ \\
\hline 10 & $\begin{array}{l}\text { Metal matrix } \\
\text { composites }\end{array}$ & G. Chadwick (UK) \\
\hline 11 & $\begin{array}{l}\text { Electroactive } \\
\text { polymers }\end{array}$ & M. Zerbi (itały) \\
\hline
\end{tabular}

Emerging Networks: Superconducting ceramics, Materials under microgravity, InP and related III-IV materials.

\section{Do You Have An Opinion?}

\section{The MRS BULLETIN wants your comments and views on issues affecting materials research.}

\author{
Send your comments to: Editor, MRS BULLETIN \\ 9800 McKnight Road \\ Pittsburgh, PA 15237 \\ Telephone (412) 367-3036 \\ Fax (412) 367-4373
}

